\title{
Boundary-value problems for weakly nonlinear ordinary differential equations
}

\section{E.N. Dancer}

In this paper, we announce results on the Dirichlet and periodic boundary-value problems for the equation $-x^{\prime \prime}(t)=g(x(t))-f(t)$ on $[0, \pi]$. We consider degenerate cases not covered by the author's earlier work.

Suppose that $g: R \rightarrow R$ is continuous and $f \in L^{1}[0, \pi]$. In this paper, we announce results on the existence of solutions of

$$
-x^{\prime \prime}(t)=g(x(t))-f(t)
$$

on $[0, \pi]$ which satisfy either

$$
x(0)=x(\pi)=0 \text { (Dirichlet boundary conditions) }
$$

or

$$
x(0)=x(\pi), x^{\prime}(0)=x^{\prime}(\pi) \text { (periodic boundary conditions). }
$$

We denote these two boundary-value problems by $\left(1_{D}\right)$ and $\left(1_{p}\right)$. We only consider cases which the results in [1] and [2] do not cover. Our results, when combined with those in [1] and [2], enable us to decide whether, for nearly all well-behaved functions $g,\left(l_{D}\right)$ (or $\left(1_{p}\right)$ ) is solvable for every $f$ in $L^{1}[0, \pi]$. (Our results suggest that some of the degenerate cases studied here are more complicated than those in [2].)

The study of the degenerate cases considered in this paper is of Received I June 1976. 
additional interest because it offers hope of progress for the corresponding problems for elliptic partial differential equations. These problems are much more difficult and most of the methods in this paper do not extend to these cases.

Throughout the paper, we assume that the limits $\mu=\lim _{y \rightarrow \infty} y^{-1} g(y)$ and $v=\lim _{y \rightarrow-\infty} y^{-1} g(y)$ exist (possibly $\pm \infty$ ). Define

$$
X_{D}=\left\{u \in C^{1}[0, \pi]: u(0)=u(\pi)=0, u^{\prime \prime} \in L^{1}[0, \pi]\right\}
$$

and define $H_{D}: X_{D}+L^{1}[0, \pi]$ by $\left(H_{D^{x}}\right)(t)=-x^{\prime \prime}(t)+g(x(t))$. Note that $X_{D}$ is a Banach space under the norm $\|x\|+\|x\| \|$, where $\|x\|$ denotes the $L^{1}$ norm of $x$. Let $R_{D}$ denote the range of $H_{D}$, that is, $R_{D}=\left\{f \in L^{l}[0, \pi]:\left(l_{D}\right)\right.$ is solvable $\} \cdot X_{p}, H_{p}$, and $R_{p}$ are defined analogously (for periodic boundary conditions).

\section{The case where $\mu$ and $v$ are finite}

We assume that the limits $I^{ \pm}=\lim _{y \rightarrow \pm \infty}\left(g(y)-\mu y^{+}+\nu y^{-}\right)$exist, where $y^{+}=\max \{y, 0\}, y^{-}=\max \{-y, 0\}$, and the limits are allowed to be infinite.

Let $\phi_{\beta}$ denote the solution of $-x^{\prime \prime}(t)=\mu(x(t))^{+}-v(x(t))^{-}$for which $\phi_{B}(0)=0$ and $\phi_{\beta}^{\prime}(0)=\beta$.

We first consider the Dirichlet problem. The results in [2] cover all cases except where $\mu \neq v$ and there exist positive integers $k$ and $l$ such that $|k-l| \leq 1$ and $k \mu^{-\frac{1}{2}}+z \nu^{-\frac{1}{2}}=1$.

THEOREM 1. Suppose that $k=2$.

(i) If $I^{+} \neq I^{-}$and at least one of $I^{+}$and $I^{-}$is infinite, $H_{D}$ is proper and onto.

(ii) If $I^{+}$and $I^{-}$are both finite, let 


$$
T=\left\{f \in L^{1}[0, \pi]: \int_{0}^{\pi} f_{+1}(t) d t \int_{0}^{\pi} f_{-1}(t) d t>0\right\},
$$

where $f_{ \pm 1}(t)=f(t) \phi_{ \pm 1}(t)-I^{+}\left(\phi_{ \pm 1}(t)\right)^{+}+I^{-}\left(\phi_{ \pm 1}(t)\right)^{-}$. Then $T$ is nonempty and $T \subseteq R_{D}$. Moreover, $R_{D}$ contains a relatively closed unbounded proper subset of $L^{\mathcal{I}}[0, \pi] \backslash \bar{T}$.

THEOREM 2. Suppose that $k-\tau=1$ and $\mu>\nu$.

(i) If $I^{+} \neq I^{-}$and either $I^{+}=-\infty$ or $I^{-}=\infty$, then $H_{D}$ is proper and onto.

(ii) If $I^{+} \neq I^{-}$and either $I^{+}=\infty$ or $I^{-}=-\infty$, then $H_{D}$ is proper, $R_{D}$ is closed, and $R_{D} \neq L^{1}[0, \pi]$.

(iii) If $I^{-}$and $I^{+}$are both finite, let

$$
\dot{T}=\left\{f \in L^{1}[0, \pi]: \int_{0}^{\pi} f_{+1}(t) d t>0\right\} .
$$

Then $T$ is non-empty and $T \subseteq R_{D}$. Moreover, $R_{D}$ contains a relatively closed unbounded proper subset of $L^{1}[0, \pi] \backslash \bar{T}$.

Natural analogues of Theorem 2 hold for the other cases in which $|k-2|=1$.

The main method used in the proof of these two theorems is a shooting argument. The following lemma is basic to this argument. For simplicity, we only state the lemma in the case where $I^{+}$is finite.

LEMMA 1. Suppose that $0 \leq t_{0} \leq t_{1} \leq \pi, u$ is a non-negative solution of (1) on $\left[t_{0}, t_{1}\right]$, and $u\left(t_{0}\right)=u\left(t_{1}\right)=0$. There exists a $K>0$ and $\varepsilon:(0, \infty) \rightarrow(0, \infty)$ with $\varepsilon(r) \rightarrow 0$ as $r \rightarrow \infty$ such that, if $u\left(t_{0}\right)=\alpha>K$, then

$$
\left|t_{1}-t_{0}-\frac{\pi}{\sqrt{\mu}}+\alpha^{-1} \int_{t_{0}}^{t_{1}}\left(I^{+}-f(s)\right) \phi_{+1}\left(s-t_{0}\right) d s\right| \leq \alpha^{-1} \varepsilon(\alpha) .
$$

Moreover, for fixed $g, \varepsilon$ and $k$ depend only on $\|f\|$. 
To prove that $L^{1}[0, \pi] \backslash \bar{T} \nsubseteq R_{D}$ in Theorem $I$ (ii), we first use a similar argument to that in the proof of Proposition l (i) in [2] to prove the result when $g$ is positive homogeneous. We then use a similar argument to that in the proof of Theorem 2 in [2] to deduce the general case. In the positive homogeneous case, we prove that $R_{D} \mid \bar{T}$ is non-empty by using the inequalities obtained by taking the scalar product of (1) with $\phi_{+1}$ (and $\phi_{-1}$ ) to show that $H_{D}(u) k \bar{T}$ if $u$ is a suitable function with small support. As before, it can then be deduced that $R_{D} \backslash \bar{T}$ is nonempty in the general case. Similar arguments are used in the proof of Theorem 2 (iii).

We now consider the periodic boundary-value problem. The only case not covered by the results in [2] is where $\mu \neq \nu$ and there is a positive integer $k$ such that $k \mu^{-\frac{3}{2}}+k \nu^{-\frac{3}{2}}=1$.

THEOREM 3. (i) If at least one of $I^{+}$and $I^{-}$is infinite and $I^{-} \neq I^{+}$then $H_{p}$ is proper and onto.

(ii) If both $I^{-}$and $I^{+}$are finite, let

$$
\begin{gathered}
F(\theta)=\int_{0}^{\pi} f(t)_{+1}(t+\theta) d t-2 k\left(\mu^{-1} I^{+}-\nu^{-1} I^{-}\right), \\
T=\left\{f \in L^{1}[0, \pi]: F(\theta) \neq 0 \text { for every } \theta \text { in }[0, \pi]\right\},
\end{gathered}
$$

and

$$
S=\left\{f \in L^{1}[0, \pi]:(F(\theta))^{2}+\left(F^{\prime}(\theta)\right)^{2}>0 \text { for every } \theta \text { in }[0, \pi]\right\} .
$$

Then $T$ is non-empty, $T \subseteq R_{p}$, and $\left(R_{p} \cap S\right) \backslash T$ is a relatively closed non-empty proper subset of $S \backslash T$.

The proof of this uses similar ideas to those in the proof of Theorem 1. However, to prove $f \in R_{p}$, we firstly use the results in [2] to solve the equation when $g$ is replaced by $g_{n}$ (where $g_{n}(y)=g(y) \pm n^{-1} y^{-}$). We next use a variant of Lemma 1 to obtain a uniform bound for these solutions and then pass to the limit. (It seems possible that this type of argument can be used in more general situations.) 
The methods of this section can be used to give an alternative proof of Theorem 8 in [2] for equation $\left(I_{D}\right)$ and $i$ ts natural analogue for $\left(l_{p}\right)$. Thus we have an alternative proof of the main result in Lazer and Leach [5].

\section{The case where exactly one of $\mu$ and $\nu$ is finite}

In this section we consider the case where $\mu=\infty$ and $\nu=i^{2}$ where $i$ is a positive integer for $\left(1_{D}\right)$ and a non-negative integer for $\left(1_{p}\right)$. The case where $\mu=i^{2}$ and $\nu=\infty$ is similar. The remaining cases are covered by the results of [2]. We assume that $I^{-}=\lim _{y^{\rightarrow-\infty}} g_{i}(y)$ exists (where $g_{i}(y)=g(y)-i^{2} y$ ) and that there exists an $M>0$ such that $g(y)+M(y-x)+M \geq g(x)$ if $y \geq x \geq M$. If $I^{-}=-\infty$, we also assume that for every $\varepsilon>0$ there exist $N_{1}, N_{2}>0$ such that $g_{i}(y) \leq(1-\varepsilon) g_{i}(x)$ if $y \leq x \leq-N_{1}$ and $g_{i}(y) \geq(1+\varepsilon) g_{i}(x)$ if $N_{2} x \leq y \leq x \leq-N_{1}$.

We first consider the Dirichlet problem. If $I^{-}=-\infty$ and $i>0$, define

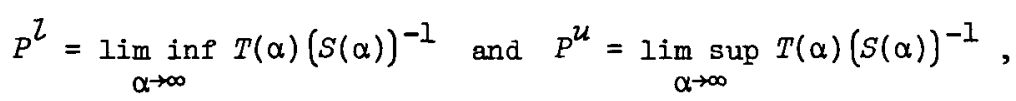

where

$$
S(\alpha)=-(i \alpha)^{-1} \int_{0}^{i^{-1} \pi} g_{i}\left(-\alpha i^{-1} \sin i t\right) \sin i t d t
$$

and $T(\alpha)$ is the first positive zero of the solution of $-x^{\prime \prime}(t)=g(x(t))$, $x(0)=0, x^{\prime}(0)=\alpha$.

THEOREM 4. (i) If $i=1$ and $I^{-}$is finite, then

$$
\left\{f \in L^{1}[0, \pi]: \int_{0}^{\pi} f(t) \sin t d t>2 I^{-}\right\} \subseteq R_{D} .
$$

Moreover, if $g(y)-y>I^{-}$for all $y$, equality holds. 
(ii) Suppose that

(a) $i>1$ and $I^{-}>-\infty$, or

(b) $i=1$ and $I^{-}=\infty$, or

(c) $i=1, I^{-}=-\infty$ and $P^{u}<\frac{1}{2}$, or

(d) $i>1, I^{-}=-\infty$ and either $P^{2}>i(i-1)^{-1}$ or $P^{u}<i(i+1)^{-1}$.

Then $H_{D}$ is proper, $R_{D}$ is closed, and $R_{D} \neq L^{1}[0, \pi]$.

(iii) Suppose that

(a) $i=1, I^{-}=-\infty$ and either $P^{2}>1$ or $\frac{1}{2}<P^{2} \leq P^{\mathcal{U}}<1$, or

(b) $i>1, I^{-}=-\infty$ and either $i(i+1)^{-1}<P^{2} \leq P^{\mathcal{U}}<1$ or $1<P^{2} \leq P^{u}<i(i-1)^{-1}$.

Then $H_{D}$ is proper and onto.

Much of the proof of this theorem is similar to the proof of Theorem 1. We use Lemma 1 and the following lemma.

LEMMA 2. Suppose that $y\left(w_{0}\right)=y\left(w_{1}\right)=0$ and $y$ is a non-negative solution of (1) on $\left[w_{0}, w_{1}\right]$. There exist $k_{1}>0$ and $\varepsilon:(0, \infty) \rightarrow(0, \infty)$ with $\varepsilon(r) \rightarrow 0$ as $r \rightarrow \infty$ such that, if $y\left(w_{0}\right)=\alpha>K_{1}$, then $\left|w_{1}-w_{0}-T(\alpha)\right| \leq \varepsilon(\alpha) T(\alpha)$. Moreover, for given $g$, $K_{1}$ and $\varepsilon$ depend only on $\|f\|$.

The other idea in the proof of Theorem 4 (ii) is to use Lemmas 1 and 2 and a limit argument to show that, if $H_{D}$ were onto, then $\left(I_{D}\right)$ would have a generalized solution when $f=c \delta_{a}$. Here $\delta_{a}$ is the Dirac delta function with support at $a \equiv \pi-(4 i)^{-1} \pi$. We can use comparison arguments to show that, if $|c|$ is large and $c$ has suitable sign, then $\left(I_{D}\right)$ is not solvable for this $f$. Thus $H_{D}$ is not onto. 
Now consider the periodic boundary-value problem.

THEOREM 5. (i) If $i=0,\left(1_{p}\right)$ is solvable if $\int_{0}^{\pi} f(t) d t>\pi I^{-}$. This condition is also necessary if $I^{-}$is finite and $g(y)>I^{*}$ for all $y$.

(ii) Suppose that $i \geq 1$ and

(a) $I^{-}>-\infty$ or

(b) $I^{-}=-\infty$ and either $P^{u}<1$ or $P^{2}>1$.

Then $H_{p}$ is proper and onto.

The proof of this result is similar to the proof of Theorem 3 .

It is possible to construct examples to show that, if $i \geq 1$ and the conditions on $P^{\mathcal{L}}$ and $P^{\mathcal{U}}$ in Theorem 4 (Theorem 5) do not hold, then $\left(H_{D}\right)^{-1}(0) \quad\left(\left(H_{p}\right)^{-1}(0)\right)$ may be unbounded.

The cases where $\mu$ and $\nu$ are both infinite are covered by the results in [2], Ehrmann [3], and Fučik and Lovicar [4].

As the full proofs are long and complicated, the author does not at present plan to publish them, but will reconsider this if he has sufficient evidence of interest in them. They are, however, freely available to those who request them from the author.

\section{References}

[1] E.N. Dancer, "On a nonlinear elliptic boundary-value problem", Buzz. Austral. Math. Soc. 12 (1975), 399-405.

[2] E.N. Dancer, "On the Dirichlet problem for weakly nonlinear elliptic partial differential equations", Proc. Roy. Soc. Edinburgh Sect. $A$ (to appear).

[3] Hans Ehrmann, "Über die Existenz der Lösungen von Randwertaufgaben bei gewöhnlichen nichtlinearen Differentialgleichungen zweiter Ordnung", Math. Ann. 134 (1957), 167-194. 
[4] Svatopluk Fučík and Vladimir Lovicar, "Periodic solutions of the equation $x^{\prime \prime}(t)+g(x(t))=p(t) "$, Časopis Pěst. Mat. 100 (1975), 160-175.

[5] A.C. Lazer and D.E. Leach, "Bounded perturbations of forced harmonic oscillators at resonance", Ann. Mat. Pura App 2. 82 (1969), 49-68.

Department of Mathematics,

University of New England,

Armidale,

New South Wales. 